\title{
Analysis of Effect of Phase Emor Sources of Polarization Components in Incoherent Triangular Holography
}

\author{
Soo-Gil Kim* \\ Department of Digital Control Engineering, Hoseo University, \\ San 29-1, Sechul-ri, Baebang-eup, Asan 336-795, Korea
}

(Received May 10, 2012 : revised June 29, 2012 : accepted July 17, 2012)

\begin{abstract}
We derive the point-spread function of the reconstructed image from a point-source complex hologram, which includes phase error caused by polarization components, in the longitudinal direction of the pointspread function and analyze the effect of the error sources of polarization components having influence on image reconstruction of a point-source complex hologram in incoherent triangular holography.

Keywords: Modified triangular interferometer, Phase error, Reconstruction, Complex hologram, Pointspread function

OCIS codes : (090.0090) Holography; (090.2880) Holographic interferometry
\end{abstract}

\section{INTRODUCTION}

Incoherent holography that is not affected by the coherence length has been considered a valuable technique for 3-D imaging in that incoherent light sources can be used. Several schemes have been presented for incoherent recording. Lohmann [1] and Cochran [2] suggested an interferometric arrangement for obtaining interference patterns from an object illuminated by an extended source. Although incoherent holography is an attractive technique, it has several drawbacks. One of the main disadvantages is that the ratio of the bias to the spatially varying intensity at the hologram plane builds up rapidly as the number of points in the object that contribute to the intensity pattern increases. Another disadvantage is that, when incoherent on-axis holograms are reconstructed, twin images appear simultaneously, and they cannot be separated. Therefore, the bias and twin images degrade the contrast of the reconstructed image.

To reduce the bias level, Kozma and Massey [3] suggested a modulation method for separating the spatially varying part from the bias. Mugnier and Sirat [4] suggested the use of a liquid-crystal light valve and a mask to resolve the bias and twin images. Recently, Poon et al.[ 5] proposed a technique that can simultaneously obtain sine and cosine Fresnel holograms to remove twin images. In previous work, we presented a new passive-device system [6], based on a triangular interferometer [2], that removes the bias and twin images using a phase-shifting technique [7, 8]. However, the quality of the reconstructed image in incoherent triangular holography is also affected by the phase error in addition to the bias and the twin images of a complex hologram. The phase error of the hologram in incoherent triangular holography is caused by imperfection of a wave plate, the azimuth angle error of a wave plate, and the azimuth angle error of a linear polarizer. [9] In this paper, we derive the point-spread function of the reconstructed image from a point-source complex hologram, which includes phase error caused by polarization components, in the longitudinal direction of the point-spread function and analyze the effect of the error sources of polarization components on the corresponding intensity of reconstructed image about the phase retardation error of a wave plate, the azimuth angle error of a wave plate, and the azimuth angle error of a linear polarizer.

\section{PRINCIPLES OF INCOHERENT TRIANGULAR HOLOGRAPHY}

In the proposed modification of the triangular interferometer, we adjust the relative phase between two optical waves that travel the triangular interferometer in clockwise (cw) and counterclockwise (ccw) directions. This is done

\footnotetext{
*Corresponding author: sgkim@hoseo.edu

Color versions of one or more of the figures in this paper are available online.
} 


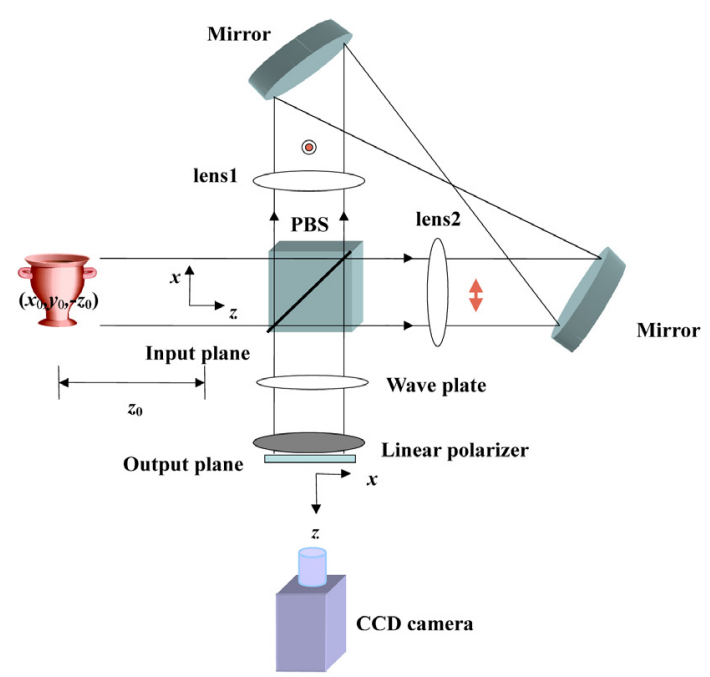

FIG. 1. Modified triangular interferometer.

by use of a polarizing beam splitter (PBS), a linear polarizer (LP), and a $N 4$ wave plate (WP). Figure 1 shows the proposed system obtained with a modified triangular interferometer (MTI). Lens 1 and lens 2 have focal lengths of $f_{1}$ and $f_{2}$, respectively. If the linear polarizer and the wave plate are removed and the polarizing beam splitter is replaced with an ordinary beam splitter, the system is the same as the Cochran triangular interferometer [2].

Both the input plane and the output plane are located at common focal planes of lens1 and lens2. In Fig. 1 we consider a single point of an incoherent object whose coordinate is given by $\left(\mathrm{x}_{0}, \mathrm{y}_{0},-\mathrm{z}_{0}\right)$, where $\mathrm{z}_{0}$ is positive $\left(\mathrm{z}_{0}\right.$ is the distance from the object to the input plane). The complex amplitudes in front of a wave plate of the light that travels $\mathrm{cw}$ and ccw, respectively, are given by

$$
\begin{aligned}
& p_{c w}(x, y)=i \frac{k}{2 \sqrt{2} \pi z_{0}} \exp \left\{-i \frac{k}{2 z_{0}}\left[\left(\alpha x-x_{0}\right)^{2}+\left(\alpha y-y_{0}\right)^{2}\right]\right\}=a \exp \left(-i \theta_{c w}\right), \\
& p_{c c w}(x, y)=i \frac{k}{2 \sqrt{2} \pi z_{0}} \exp \left\{-i \frac{k}{2 z_{0}}\left[\left(\beta x-x_{0}\right)^{2}+\left(\beta y-y_{0}\right)^{2}\right]\right\}=b \exp \left(-i \theta_{c c w}\right),
\end{aligned}
$$

where $k$ is the wave number, $z_{0}$ is the distance from the object to the input plane, and $\alpha \equiv-f_{1} / f_{2}, \beta \equiv-f_{2} / f_{1}$. By adjusting the relative phase between two optical waves using the linear polarizer and the wave plate, we can obtain the four intensities at the output plane as listed in Table 1:

$$
\begin{aligned}
& \phi(x, y)=\theta_{c w}(x, y)-\theta_{c c w}(x, y)=\left(k / 2 z_{1}\right)\left\{\left(x-x_{1}\right)^{2}+\left(y-y_{1}\right)^{2}-\left(x_{1}^{2}+y_{1}^{2}\right)\right\}, \\
& x_{1}=x_{0} /(\alpha+\beta), \\
& y_{1}=y_{0} /(\alpha+\beta), \\
& z_{1}=z_{0} /\left(\alpha^{2}-\beta^{2}\right) .
\end{aligned}
$$

TABLE 1 . Intensity patterns by combination of a wave plate and a linear polarizer

\begin{tabular}{c|c|c}
\hline $\begin{array}{c}\text { Azimuth angle of a } \\
\text { linear polarizer } \\
\text { (degree) }\end{array}$ & $\begin{array}{c}\text { Phase retardation } \\
\text { of a wave plate }\end{array}$ & $\begin{array}{c}\text { Point-Spread } \\
\text { Function }\end{array}$ \\
\hline 0 & $\Gamma=\frac{\pi}{2}$ & $\frac{1}{2}[1-\sin \phi(x, y)]$ \\
\cline { 2 - 3 } & $\Gamma=-\frac{\pi}{2}$ & $\frac{1}{2}[1+\sin \phi(x, y)]$ \\
\hline 45 & $\Gamma=\frac{\pi}{2}$ & $\frac{1}{2}[1+\cos \phi(x, y)]$ \\
\hline-45 & $\Gamma=\frac{\pi}{2}$ & $\frac{1}{2}[1-\cos \phi(x, y)]$ \\
\hline
\end{tabular}

See $[6,10]$ with respect to the above equations. By controlling four intensities either electronically or optically, we obtain the final complex hologram without bias:

$$
H(x, y)=\exp \{ \pm i \phi(x, y)\}
$$

Therefore, bias and twin images can be removed by the choice of four sequential sets of LP and WP and manipulation of the detected light intensities electronically.

\section{PHASE ERROR OF INCOHERENT TRIANGULAR HOLOGRAPHY}

The main potential sources of error in the MTI are the imperfections of the polarization elements and azimuth angle error of the polarization elements. We shall analyze the effects of these potential error sources one by one.

In Fig. 1, the Jones matrix [11] of output beam in the output plane is given by

$$
E_{\text {out }}=A\left(\varphi_{2}\right) W\left(\varphi_{1}\right) E_{\text {in }},
$$

where $E_{\text {in }}$ represents input optical wave, and $\mathrm{A}\left(\varphi_{2}\right)$ and $W\left(\varphi_{1}\right)$ represent Jones matrices of a linear polarizer and a wave plate, respectively. The Jones matrices of polarization elements are given by, respectively,

$$
\begin{aligned}
& E_{i n}=\left(\begin{array}{c}
p_{c c w} \\
p_{c w}
\end{array}\right)=\left(\begin{array}{c}
b \exp \left(-i \theta_{c c w}\right) \\
a \exp \left(-i \theta_{c w}\right)
\end{array}\right), \\
& \mathrm{A}\left(\varphi_{2}\right)=\left(\begin{array}{cc}
\cos ^{2} \varphi_{2} & (1 / 2) \sin 2 \varphi_{2} \\
(1 / 2) \sin 2 \varphi_{2} & \sin ^{2} \varphi_{2}
\end{array}\right), \\
& W\left(\varphi_{1}\right)=\left(\begin{array}{cc}
2 i \sin ^{2} \varphi_{1} \sin \frac{\Gamma}{2}+\exp \left(-i \frac{\Gamma}{2}\right) & -i \sin 2 \varphi_{1} \sin \frac{\Gamma}{2} \\
-i \sin 2 \varphi_{1} \sin \frac{\Gamma}{2} & -2 i \sin ^{2} \varphi_{1} \sin \frac{\Gamma}{2}+\exp \left(i \frac{\Gamma}{2}\right)
\end{array}\right),
\end{aligned}
$$


where $W\left(\varphi_{1}\right)$ represents the Jones matrix for $\lambda / 4$ plate. $\varphi_{2}$ and $\varphi_{1}$ represent the azimuth angle of a linear polarizer and a wave plate, respectively. $\Gamma$ represents the phase retardation of a wave plate. The intensity of Eq. (4) is given by

$$
I=\left|E_{\text {out }}\right|^{2}
$$

\subsection{Phase Enor Caused by the Imperfections of a Wave Plate}

We shall deal mainly with the errors that are introduced by imperfections in the $\lambda / 4$ plate. In this case, we assume that the azimuth angle error of the wave plate is zero. The phase error introduced by imperfections in the $\lambda / 4$ plate can be obtained from four intensity patterns as listed in Table 2, which are obtained from Eqs. (4) to (8).

From Table 2, the phase difference $\phi^{\prime}$ of optical waves $p_{c w}$ and $p_{c c w}$ is given by

$$
\tan \phi^{\prime}=\frac{I_{2}-I_{1}}{I_{3}-I_{4}}=\tan \phi \sin \Gamma
$$

where $\phi^{\prime}$ includes the error introduced by a wave plate. For a nonideal $\lambda / 4$ plate, we have

$$
\Gamma=\frac{\pi}{2}+\omega
$$

where $\omega$ is the error in the relative retardation introduced by plate. Substituting Eq. (10) into Eq. (9), we obtain

$$
\tan \phi^{\prime}=\tan \phi \cos \omega .
$$

TABLE 2 . Intensity patterns by combination of a wave plate and a linear polarizer

\begin{tabular}{c|c|c}
\hline $\begin{array}{c}\text { Azimuth angle } \\
\text { of a linear polarizer } \\
\text { (degree) }\end{array}$ & $\begin{array}{c}\text { Azimuth angle of } \\
\text { a wave plate } \\
\text { (degree) }\end{array}$ & Point-Spread Function \\
\hline 0 & $\frac{1}{2} b^{2}(1+\cos \Gamma)-a b \sin \phi \sin \Gamma$ \\
\cline { 2 - 3 } & 45 & $I_{3}=\frac{1}{2} a^{2}+\frac{1}{2} b^{2}+a b \cos \phi$ \\
\cline { 2 - 3 } & -45 & $I_{4}=\frac{1}{2} a^{2}+\frac{1}{2} b^{2}-a b \cos \phi$ \\
\hline-45 & 0 & $\frac{I_{2}}{2}=\frac{1}{2} a^{2}(1-\cos \Gamma)+$ \\
\hline & & $\frac{1}{2} b^{2}(1+\cos \Gamma)+a b \sin \phi \sin \Gamma$ \\
\hline
\end{tabular}

Since

$$
\tan \phi^{\prime}=\tan (\phi+\Delta \phi) \approx \tan \phi+\Delta \phi \sec ^{2} \phi,
$$

we can calculate the phase error that is due to the imperfections of a wave plate from Eqs. (11) and (12) as follows:

$$
\Delta \phi_{w p}=-\frac{1}{4} \omega^{2} \sin (2 \phi) .
$$

\subsection{Phase Error Caused by the Azimuth Angle Error of a Wave Plate}

In this section, we shall deal with the phase error that is introduced by the azimuth angle error in the $\lambda / 4$ plate. In this case, we assume that a wave plate is ideal and the azimuth angle errors of all polarization elements are zero except for a wave plate. The azimuth angle of a wave plate means the angle that the slow axis of a wave plate is rotated with respect to horizontal axis as shown in Fig. 2.

For $\varphi_{1}=\pi / 4$ and $\varphi_{1}=-\pi / 4$, we assume that azimuths of a wave plate are $\varphi_{1}=\pi / 4+\beta_{1}$ and $\varphi_{1}=\pi / 4+\beta_{2}$, where $\beta_{1}$ and $\beta_{2}$ represent the azimuth angle errors of a wave plate.

The phase error introduced by the azimuth angle error of a wave plate can be obtained from four intensity patterns as listed in Table 3.

By the same procedure as section 3.1, we obtain the phase error due to the azimuth angle of a wave plate as

$$
\Delta \phi_{a w p}=\beta_{1}\left(1+\sin ^{2} \phi-2 \cot 2 \beta \sin \phi\right)-\beta_{2} \cos ^{2} \phi,
$$

where $\cot 2 \beta=\left(a^{2}-b^{2}\right) / 2 a b$.

If $a=b$, then $\cot 2 \beta=0$. In this case, phase error is as follows:

$$
\Delta \phi_{\text {awp }}=\beta_{1}\left(1+\sin ^{2} \phi\right)-\beta_{2} \cos ^{2} \phi .
$$

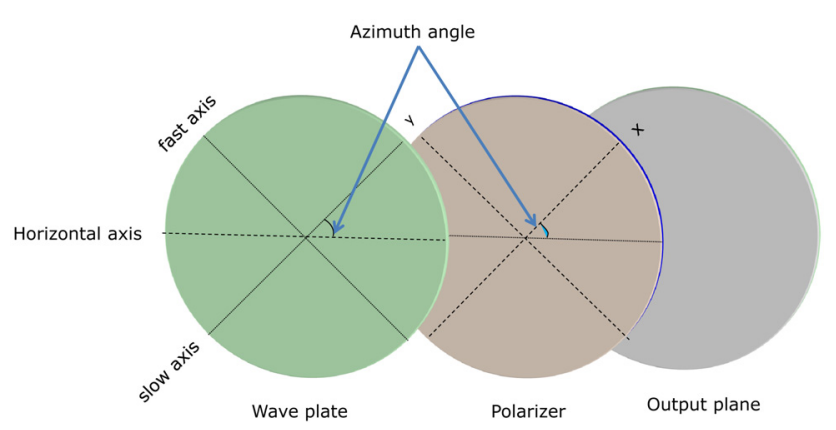

FIG. 2. Illustration for the azimuth angle of a wave plate and a linear polarizer. 
TABLE 3. Intensity patterns by combination of a wave plate and a linear polarizer considering only azimuth angle error of a wave plate

\begin{tabular}{|c|c|c|}
\hline $\begin{array}{c}\text { Azimuth angle of } \\
\text { a linear polarizer } \\
\text { (degree) }\end{array}$ & $\begin{array}{c}\text { Azimuth angle of } \\
\text { a wave plate } \\
\text { (degree) }\end{array}$ & Point-Spread Function \\
\hline \multirow{2}{*}{0} & $45+\beta_{1}$ & $\begin{array}{c}I_{1}=\frac{1}{2} a^{2}+b^{2}\left(\frac{1}{2}+2 \beta_{1}^{2}\right) \\
-a b\left(2 \beta_{1} \cos \phi+\sin \phi\right)\end{array}$ \\
\hline & $-45+\beta_{2}$ & $\begin{array}{c}I_{2}=\frac{1}{2} a^{2}+b^{2}\left(\frac{1}{2}+2 \beta_{2}^{2}\right) \\
-a b\left(2 \beta_{2} \cos \phi-\sin \phi\right)\end{array}$ \\
\hline 45 & $45+\beta_{1}$ & $\begin{array}{c}I_{3}=\frac{1}{2} a^{2}\left(1+2 \beta_{1}+2 \beta_{1}^{2}\right)+ \\
\frac{1}{2} b^{2}\left(1-2 \beta_{1}+2 \beta_{1}^{2}\right)+ \\
a b\left(\cos \phi-2 \beta_{1} \sin \phi\right)\end{array}$ \\
\hline-45 & $45+\beta_{1}$ & $\begin{array}{c}I_{4}=\frac{1}{2} a^{2}\left(1-2 \beta_{1}+2 \beta_{1}^{2}\right)+ \\
\frac{1}{2} b^{2}\left(1+2 \beta_{1}+2 \beta_{1}^{2}\right)- \\
a b\left(\cos \phi-2 \beta_{1} \sin \phi\right)\end{array}$ \\
\hline
\end{tabular}

3.3. Phase error caused by the azimuth angle error of a linear polarizer

We deal with the phase error that is introduced by the azimuth angle error in the linear polarizer. In this case, we assume that a linear polarizer is ideal and the azimuth angle errors of all polarization elements are zero except for a linear polarizer. The azimuth angle of a linear polarizer means the angle that the $\mathrm{x}$-axis of a linear polarizer is rotated with respect to horizontal axis as shown in Fig. 2.

For $\varphi_{2}=0, \varphi_{2}=\pi / 4$ and $\varphi_{1}=-\pi / 4$, we assume that azimuths of a linear polarizer including error are $\varphi_{2}=0+$ $\gamma_{1}, \quad \varphi_{2}=\pi / 4+\gamma_{2}$ and $\varphi_{2}=-\pi / 4+\gamma_{3}$, respectively, and where $\gamma_{1}, \gamma_{2}$, and $\gamma_{3}$ represent the azimuth angle errors of a linear polarizer.

The phase error introduced by the azimuth angle error of a linear polarizer can be obtained from four intensity patterns as listed in Table 4.

By the same procedure as section 3.1, we obtain the phase error caused by the azimuth angle of a linear polarizer as

$$
\Delta \phi_{a l p}=-\left(\gamma_{2}+\gamma_{3}\right) \sin ^{2} \phi
$$

\subsection{Total Phase Enor Caused by the Polarization Com- ponents}

The total phase error by polarization components is written by adding Eqs. (13), (15), and (16) giving

$$
\Delta \phi=-\frac{1}{4} \omega^{2} \sin (2 \phi)+\beta_{1}\left(1+\sin ^{2} \phi\right)-\beta_{2} \cos ^{2} \phi-\left(\gamma_{2}+\gamma_{3}\right) \sin ^{2} \phi .
$$

In the extraction of the phase term using the combination
TABLE 4. Intensity patterns by combination of a wave plate and a linear polarizer only azimuth angle error of a linear polarizer

\begin{tabular}{c|c|c}
\hline $\begin{array}{c}\text { Azimuth angle of } \\
\text { a linear polarizer } \\
\text { (degree) }\end{array}$ & $\begin{array}{c}\text { Azimuth angle of } \\
\text { a wave plate } \\
\text { (degree) }\end{array}$ & Point-Spread Function \\
\hline \multirow{2}{*}{$0+\gamma_{1}$} & 45 & $\begin{array}{c}I_{1}=\frac{1}{2}\left(a^{2}+b^{2}\right)+ \\
a b\left(2 \gamma_{1} \cos \phi-\sin \phi\right)\end{array}$ \\
\cline { 2 - 3 } & -45 & $I_{2}=\frac{1}{2}\left(a^{2}+b^{2}\right)+$ \\
& $a b\left(2 \gamma_{1} \cos \phi+\sin \phi\right)$ \\
\hline $45+\gamma_{2}$ & 45 & $I_{3}=\frac{1}{2}\left(a^{2}+b^{2}\right)+$ \\
$-45+\gamma_{2}$ & 45 & $a b\left(\cos \phi+2 \gamma_{2} \sin \phi\right)$ \\
\hline
\end{tabular}

of polarization elements, the phase error occurs. The retardation error of the commercially available wave plate makes the second-order error very small. Accordingly, the phase error in the MTI is mainly due to the azimuth angle errors of the polarization elements.

\section{EFFECT OF ERROR SOURCES OF POLARIZATION COMPONENTS ON THE IMAGE RECONSTRUCTION OF THE COMPLEX HOLOGRAM}

\subsection{Imperfections of a Wave Plate}

A point-source hologram considering the phase error of Eq. (13) due to a wave plate is described as

$$
H_{r}(x, y)=\exp \left(-i \phi_{w p}\right)
$$

where $H_{r}$ represents the complex hologram corresponding to real image and $\phi_{\wp}=\phi-\frac{1}{4} \omega^{2} \sin 2 \phi$. For simplicity, we now consider a single point-source hologram whose coordinate $\left(x_{1}, y_{1}\right)$ is given by $(0,0)$. Then $H_{r}$ becomes

$$
H_{r}(x, y)=\exp \left\langle-i\left\{\left(k / 2 z_{1}\right)\left(x^{2}+y^{2}\right)-\frac{1}{4} \omega^{2} \sin \left[\left(k / z_{1}\right)\left(x^{2}+y^{2}\right)\right]\right\}\right\rangle
$$

According to Fresnel diffraction theory, the complex amplitude is given by

$$
U(x, y, z)=\frac{i}{\lambda z} \exp (-i k z) \iint_{\xi^{2}+\eta^{2} \leq R^{2}} H_{r}(\xi, \eta) \exp \left\{-i \frac{k}{2 z}\left[(x-\xi)^{2}+(y-\eta)^{2}\right]\right\} d \xi d \eta,
$$


where $\mathrm{R}$ is the radius of the hologram size. Considering the complex amplitude in the z-axis direction, Eq. (20) becomes

$$
U(0,0, z)=\frac{i}{\lambda z} \exp (-i k z) \iint_{\xi^{2}+\eta^{2} \leq R^{2}} H_{r}(\xi, \eta) \exp \left[-i \frac{k}{2 z}\left(\xi^{2}+\eta^{2}\right)\right] d \xi d \eta .
$$

If there is negligible overlap of the various diffracted terms, the corresponding intensity of the reconstructed image of point-source hologram along the z-axis direction becomes

$$
I(0,0, z)=\frac{k^{2} R^{4}}{4 z^{2}} \sum_{m=-\infty}^{\infty} J_{m}{ }^{2}\left(-\frac{1}{4} \omega^{2}\right) \sin c^{2}\left\{\frac{k}{4}\left(\frac{1}{z_{1}}-\frac{1}{z}+\frac{2 m}{z_{1}}\right) R^{2}\right\},
$$

where $J_{x}(\cdot)$ is a Bessel function of the first kind, order $\mathrm{x}$.

The intensity patterns corresponding to Eq. (22) are plotted together in Fig. 3; the phase retardation errors of a wave plate(i.e. $\omega$ ) for $2 \pi / 300,2 \pi / 150,2 \pi / 50,2 \pi / 30$, $2 \pi / 15$, and $2 \pi / 8$ were considered. Also, hologram size, wavelength and the distance from the object to the input plane were set to $0.05 \mathrm{~m}, 632.8 \mathrm{~nm}$ and $0.3 \mathrm{~m}$, respectively, and $\alpha^{2}-\beta^{2}$ was set to unity.

Figure 3 (a) and (b) represent the 3-D plot of normalized intensity for the phase retardation errors caused by the

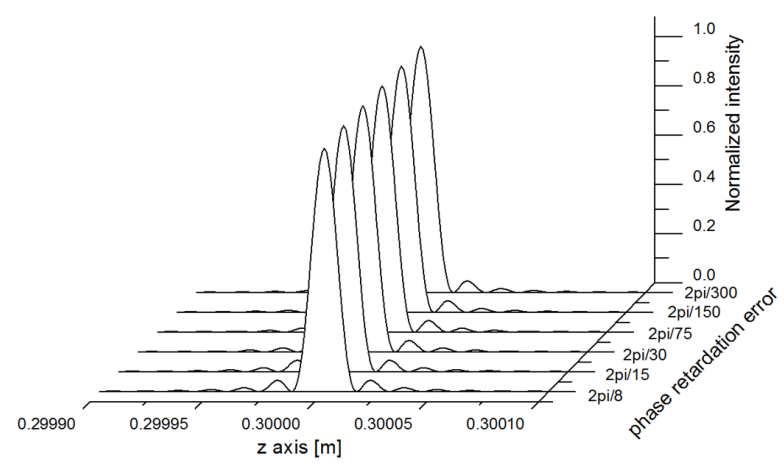

(a)

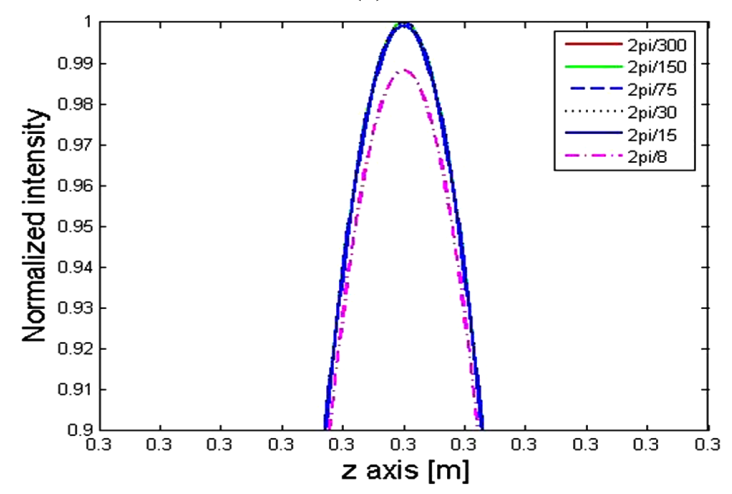

(b)

FIG. 3. Normalized intensity of reconstructed image of a point-source hologram for phase error due to the imperfections of wave plate. imperfections of a wave plate and the front view for the normalized intensity beyond 0.9 in Fig. 3 (a), respectively. As shown in Fig. 3, as phase retardation error increases, the intensity of reconstructed image has very little change. When the phase retardation errors range from $2 \pi / 300$ to $2 \pi / 8$, the normalized intensity of reconstructed image is down by about $1.2 \%$. This means that the reconstructed image of complex hologram created by using the commercially available wave plate is not influenced by the phase retardation error of a wave plate.

\subsection{Azimuth Angle Error of a Wave Plate}

By the same procedure as section 4.1, we obtain the corresponding intensity of the reconstructed image of point-source hologram along the z-axis direction using the phase error of Eq. (14) as

$$
\begin{aligned}
I(0,0, z) & =\frac{k^{2} R^{4}}{4 z^{2}} \sum_{q=-\infty}^{\infty} J_{q}{ }^{2}\left\{-\frac{1}{2}\left(\beta_{1}+\beta_{2}\right)\right\} \\
& \times \sum_{m=-\infty}^{\infty} J_{m}{ }^{2}\left\{-2 \beta_{1} \cot 2 \beta\right) \operatorname{sinc}^{2}\left\{\frac{k}{4}\left(\frac{1}{z_{1}}-\frac{1}{z}+\frac{2 q}{z_{1}}+\frac{m}{z_{1}}\right) R^{2}\right\} .
\end{aligned}
$$

The intensity patterns corresponding to Eq. (23) are plotted together in Fig. 4; the azimuth angle errors of a wave plate(i.e. $\beta_{1}+\beta_{2}$ ) for $2 \pi / 300,2 \pi / 150,2 \pi / 50,2 \pi / 30$, $2 \pi / 15$, and $2 \pi / 8$ were considered. Figure 4 (a) and (b)

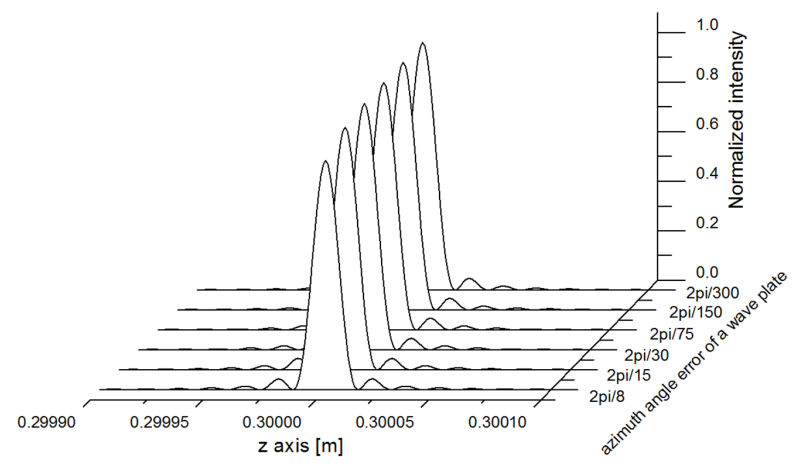

(a)

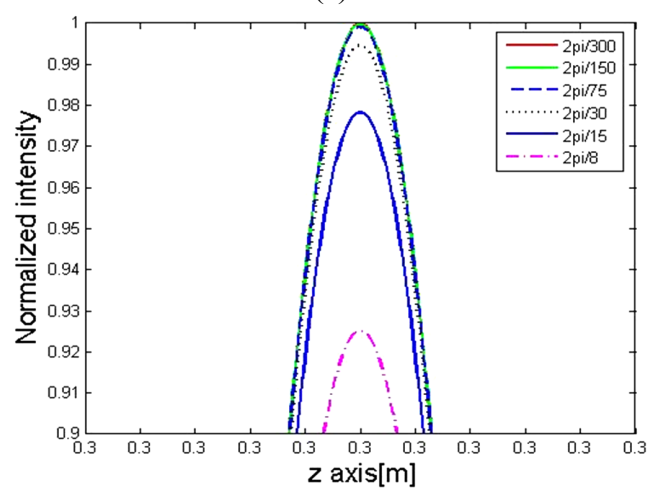

(b)

FIG. 4. Normalized intensity of reconstructed image of a point-source hologram for phase error due to the azimuth angle error of a wave plate. 
represent 3-D plot of normalized intensity for the azimuth angle error of a wave plate and the front view for the normalized intensity beyond 0.9 in Fig. 4 (a), respectively.

As shown in Fig. 4, as azimuth angle error increases, the intensity of the reconstructed image decreases. When the azimuth angle errors of a wave plate range from $2 \pi$ $/ 300$ to $2 \pi / 8$, the normalized intensity of the reconstructed image is down by about $7.5 \%$. Decrease of the normalized intensity caused by azimuth angle error of a wave plate is nearly 7 times that caused by imperfections of a wave plate. This means that the reconstructed image of the complex hologram is influenced by not imperfection of a wave plate but the azimuth angle error of a wave plate.

\subsection{Azimuth Angle Error of a Linear Polarizer}

By the same procedure as section 4.1, we obtain the corresponding intensity of the reconstructed image of the point-source hologram along the $\mathrm{z}$-axis direction using the phase error of Eq. (15) as

$$
I(0,0, z)=\frac{k^{2} R^{4}}{4 z^{2}} \sum_{q=-\infty}^{\infty} J_{q}^{2}\left\{\frac{1}{2}\left(\gamma_{2}+\gamma_{3}\right)\right\} \sin c^{2}\left\{\frac{k}{4}\left(\frac{1}{z_{1}}-\frac{1}{z}+\frac{2 q}{z_{1}}\right) R^{2}\right\}
$$

The intensity patterns corresponding to Eq. (24) are plotted together in Fig. 5; the azimuth angle errors of a linear polarizer (i.e. $\gamma_{2}+\gamma_{3}$ ) for $2 \pi / 300,2 \pi / 150,2 \pi / 50$, $2 \pi / 30,2 \pi / 15$, and $2 \pi / 8$ were considered. Figure 5 (a) and (b) represent 3-D plot of normalized intensity for the azimuth angle error of a linear polarizer and the front view for the normalized intensity beyond 0.9 in Fig. 5 (a), respectively.

As shown in Fig. 5, as azimuth angle error increases, the intensity of the reconstructed image decreases. When the azimuth angle errors of the linear polarizer range from $2 \pi / 300$ to $2 \pi / 8$, the normalized intensity of the reconstructed image is down by about $7.5 \%$. Decrease of the normalized intensity caused by azimuth angle error of a linear polarizer is nearly 7 times that caused by imperfections of a wave plate. This means that the reconstructed image of the complex hologram is influenced by the azimuth angle error of a linear polarizer. Figure 4 and 5 are pretty similar. From the results, we conclude that the azimuth angle error of polarization components has a similar influence on image reconstruction of the complex hologram.

\subsection{Total Emor}

By the same procedure as section 4.1, we obtain the corresponding intensity of the reconstructed image of the point-source hologram along the z-axis direction using the phase error of Eq. (16) as

$$
\begin{aligned}
I(0,0, z)= & \frac{k^{2} R^{4}}{4 z^{2}} \sum_{m=-\infty}^{\infty} J_{m}{ }^{2}\left(-\frac{1}{4} \omega^{2}\right) \sum_{q=-\infty}^{\infty} J_{q}{ }^{2}\left\{-\frac{1}{2}\left(\beta_{1}+\beta_{2}-\gamma_{2}-\gamma_{3}\right)\right\} \\
& \times \operatorname{sinc}^{2}\left\{\frac{k}{4}\left(\frac{1}{z_{1}}-\frac{1}{z}+\frac{2 m}{z_{1}}+\frac{2 q}{z_{1}}\right) R^{2}\right\}
\end{aligned}
$$

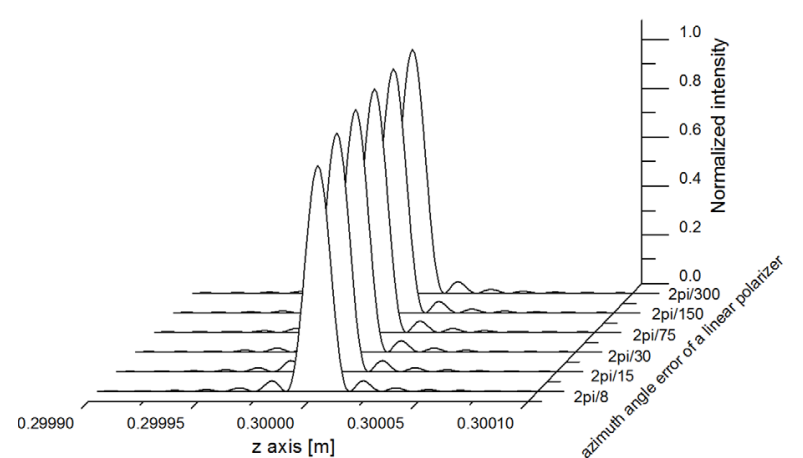

(a)

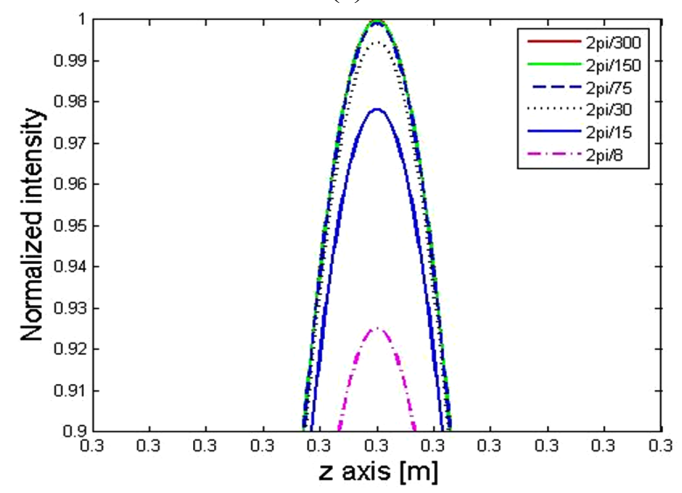

(b)

FIG. 5. Normalized intensity of reconstructed image of a point-source hologram for phase error due to the azimuth angle error of a linear polarizer.

where $J_{x}(\cdot)$ is a Bessel function of the first kind, order $x$. For the case that the total phase error is zero, intensity is equal to the result of [8] and the intensity of the reconstructed image is sinc function and is maximum at $\mathrm{z}_{1}$.

The intensity patterns corresponding to Eq. (25) are plotted together in Fig. 6, in which the phase retardation error of a wave plate(i.e. $\omega$ ) was set to $2 \pi / 300$ and six error sums(i.e. $\beta_{1}+\beta_{2}-\gamma_{2}-\gamma_{3}$ ) for $2 \pi / 300,2 \pi / 150,2 \pi / 50,2 \pi / 30$, $2 \pi / 15$, and $2 \pi / 8$ were considered.

Figure 6 (a) and (b) represent 3-D plot for the total error sum and the front view for the normalized intensity beyond 0.9 in Fig. 6 (a), respectively. As shown in Fig. 6, as total error sum increases, the intensity of the reconstructed image decreases. When the total errors range from $2 \pi / 300$ to $2 \pi / 8$, the normalized intensity is down by about $7.5 \%$.

Considering the Rayleigh criterion, we can conclude that the longitudinal resolution of the reconstructed image decreases as the total phase error increases because the total phase error is proportional to the error sum. From the results, it can be seen that the resolving power of an incoherent triangular holography in the longitudinal direction is inversely proportional to the total error. In conclusion, the minimum separation of a three-dimensional object that can be resolved by the incoherent triangular interferometer is proportional to the phase error of the polarization elements. 


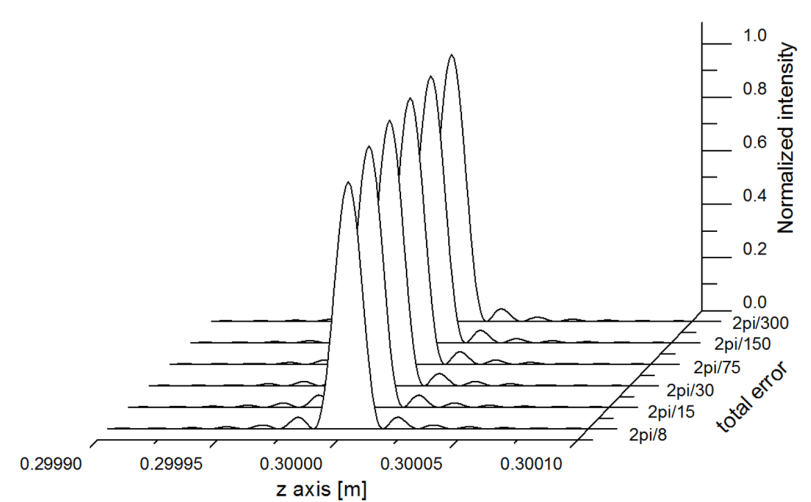

(a)

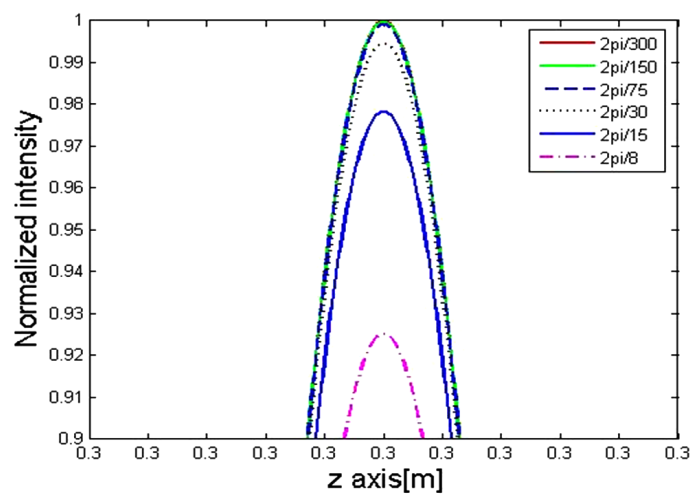

(b)

FIG. 6. Normalized intensity of reconstructed image of a point-source hologram for total phase error by polarization components.

\section{CONCLUSION}

We derived the point-spread function of the reconstructed image from a point-source complex hologram, which includes phase error caused by polarization components, in the longitudinal direction of the point-spread function and analyzed the effect of the error sources of polarization components on the corresponding intensity of reconstructed image about the phase retardation error of a wave plate, the azimuth angle error of a wave plate, and the azimuth angle error of a linear polarizer.

As phase retardation error of a wave plate increases, the intensity of the reconstructed image changes very little. On the other hand, as azimuth angle error of polarization components increases, the intensity of the reconstructed image decreases.

From the results, the commercially available wave plates don't have an influence on image reconstruction of the complex hologram. The quality of the reconstructed image in incoherent triangular holography is affected by the azimuth angle errors of the polarization elements, which have a similar influence on image reconstruction of the complex hologram.

\section{ACKNOWLEDGMENT}

This research was supported by the Academic Research fund of Hoseo University in 2009.(2009-0530)

\section{REFERENCES}

1. A. W. Lohmann, "Wavefront reconstruction for incoherent objects," J. Opt. Soc. Am. 55, 1555-1556 (1965).

2. G. Cochran, "New method of making Fresnel transforms with incoherent light," J. Opt. Soc. Am. 56, 1513-1517 (1966).

3. A. Kozma and N. Massey, "Bias level reduction of incoherent holograms," Appl. Opt. 8, 393-397 (1969).

4. L. M. Mugnier and G. Y. Sirat, "On-axis conoscopic holography without a conjugate image," Opt. Lett. 17, 294-296 (1992).

5. T.-C. Poon, T. Kim, G. Indebetouw, B. W. Schilling, M. H. Wu, K. Shinoda, and Y. Suzuki, "Twin-image elimination experiments for three-dimensional images in optical scanning holography," Opt. Lett. 25, 215-217 (2000).

6. S.-G. Kim, B. Lee, and E.-S. Kim, "Removal of bias and the conjugate image in incoherent on-axis triangular holography and real-time reconstruction of the complex hologram," Appl. Opt. 36, 4784-4791 (1997).

7. W. Steinchen and L. Yang, Digital Shearography (SPIE Press, USA, 2003), Chapter 4.

8. S.-H. Jeon and S.-K. Gil, "QPSK modulation based optical image cryptosystem using phase-shifting digital holography," J. Opt. Soc. Korea 14, 97-103 (2010).

9. S.-G. Kim and J. Ryeom, "Phase error analysis of incoherent triangular holography," Appl. Opt. 48, H231-H237 (2009).

10. S.-G. Kim, B. Lee, E.-S. Kim, and C.-W. Yi, "Resolution analysis of incoherent triangular holography," Appl. Opt. 40, 4672-4678 (2001).

11. A. Yariv and P. Yeh, Optical Waves in Crystals (Wiley, New York, USA, 1984), Chapter 5. 